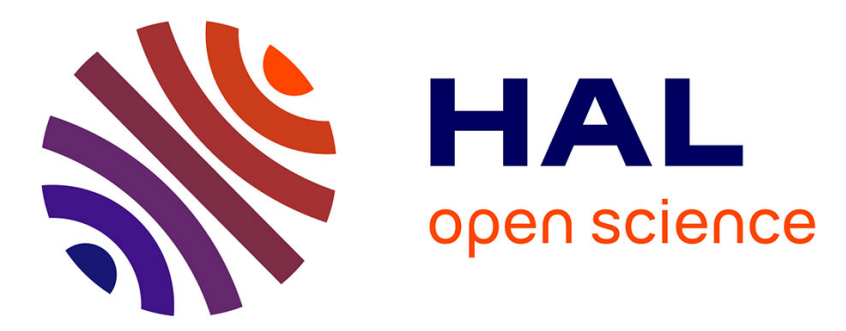

\title{
Study of electromagnetic diffraction by curved strip gratings by use of the C-method
}

\author{
B. Guizal, G. Granet
}

\section{To cite this version:}

B. Guizal, G. Granet. Study of electromagnetic diffraction by curved strip gratings by use of the C-method. Journal of the Optical Society of America. A Optics, Image Science, and Vision, 2007, 24 (3), pp.669-674. 10.1364/JOSAA.24.000669 . hal-00255748

\section{HAL Id: hal-00255748 \\ https://hal.science/hal-00255748}

Submitted on 22 Apr 2021

HAL is a multi-disciplinary open access archive for the deposit and dissemination of scientific research documents, whether they are published or not. The documents may come from teaching and research institutions in France or abroad, or from public or private research centers.
L'archive ouverte pluridisciplinaire HAL, est destinée au dépôt et à la diffusion de documents scientifiques de niveau recherche, publiés ou non, émanant des établissements d'enseignement et de recherche français ou étrangers, des laboratoires publics ou privés. 


\title{
Study of electromagnetic diffraction by curved strip gratings by use of the C-method
}

\author{
B. Guizal \\ Départment d'Optique P. M. Duffieux, Institut Franche-Comté Electronique, Mécanique, Thermique et \\ Optique-Sciences et Technologies, UMR 6174 du CNRS, 16, Route de Gray, 25030 Besanon Cedex, France
}

\section{G. Granet}

Laboratoire des Sciences et Matériaux pour l'Electronique, et d'Automatique, UMR CNRS 6602, Université Blaise Pascal Les Cézeaux, 24 avenue des Landais, 63177 Aubière Cedex, France

\begin{abstract}
The C-method is one of the most efficient and versatile methods designed for diffraction gratings. Its strength lies in the use of a coordinate system in which the surface of the grating coincides with a coordinate surface. The result is a great simplification in writing the boundary conditions. We exploit this simplification to treat the problem of diffraction from curved strip gratings, and we use the combined boundary conditions method that has been introduced for planar strip gratings and proved to be very efficient.
\end{abstract}

\section{INTRODUCTION}

Diffraction from strip gratings is a well-known problem that has been extensively studied in the past. These structures have been used to model photolithographic masks as well as optical or microwave filters or frequency selective surfaces. Although some rigorous methods do exist that can model more realistic situations, i.e., strips with finite conductivity and non-null thickness, the perfect (in the sense perfectly conducting and infinitely thin) strip grating approach remains interesting and can even be competitive when one is dealing with highly conducting materials with a sufficiently small thickness compared to the wavelength, as can be the case of noble metals in the terahertz domain. One of the most versatile and efficient methods designed for perfectly conducting strip gratings is the combined boundary conditions method (CBCM). ${ }^{1}$ The key feature of this differential approach is that it combines the continuity equations of the electric and the magnetic fields in a unified equation that holds over one full period. Thus all the continuity equations can be projected on the Fourier basis permitting simple calculations. This method has been applied to strip gratings ${ }^{1}$ as well as bulk ones ${ }^{2}$ and adapted successfully to aperiodic strips gratings ${ }^{3}$ and to circular geometry. ${ }^{4}$

On the other hand, the C-method ${ }^{5}$ is one of the most elegant and efficient methods introduced for surface relief diffraction gratings. Its strength lies in the use of a coordinate system in which the surface of the grating coincides with a coordinate surface. The result is a great simplification in writing the boundary conditions. Since its introduction in 1982, this approach has been generalized to multilayered diffraction gratings ${ }^{6}$ and to crossed gratings. ${ }^{7}$ In this work, we apply the C-method to the study of curved diffraction gratings.
In Sections 2 and 3 the key steps of the C-method and the CBCM are outlined in order to give some selfconsistency to the paper. Sections 4 and 5 are dedicated to the writing of the boundary conditions through the concept of the CBCM. Finally, some numerical examples are given to illustrate the effectiveness of this new approach.

\section{THEORY}

The structure under study is depicted in Fig. 1. It consists of a one-dimensional grating separating two dielectric homogeneous media and over which is deposited an infinitely thin perfectly conducting grating of the same shape and period. The surface of the grating is invariant along the $z$ direction, and we assume that its cross section (i.e., the shape of the grating) can be described by a function, $a(x)$. It is known from previous work ${ }^{5}$ that it is possible to introduce a new coordinate system such that the surface of the grating coincides with a coordinate surface. Such a system, called the translation coordinate system, can be defined from the Cartesian coordinates as follows:

$$
\begin{aligned}
& x^{1}=x, \\
& x^{2}=u=y-a(x), \\
& x^{3}=z .
\end{aligned}
$$

In the new coordinate system $(x, u, z)$, the surface of the grating is exactly that of equation $u=0$. This makes the boundary conditions much simpler to write, as will be recalled later. In this coordinate transformation, the metric tensor writes 


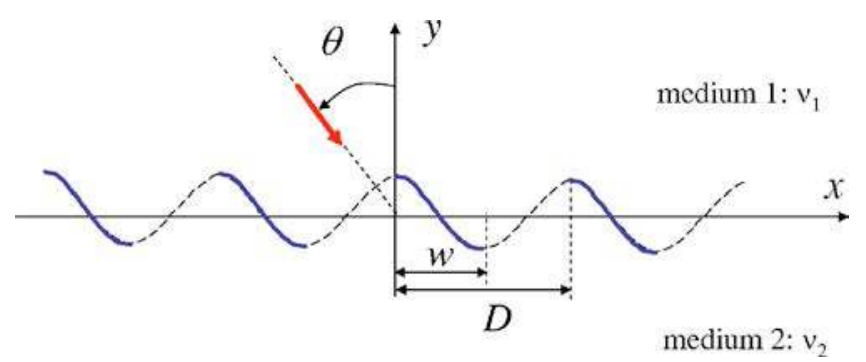

Fig. 1. (Color online) Geometry of the problem.

$$
\begin{aligned}
\left(g^{l m}\right) & =\left[\begin{array}{lll}
g^{11} & g^{12} & g^{13} \\
g^{21} & g^{22} & g^{23} \\
g^{31} & g^{32} & g^{33}
\end{array}\right]=\left[\begin{array}{ccc}
1 & -\dot{a} & 0 \\
-\dot{a} & 1+\dot{a}^{2} & 0 \\
0 & 0 & 1
\end{array}\right], \\
\dot{a} & =\frac{\mathrm{d} a(x)}{\mathrm{d} x} .
\end{aligned}
$$

One can remark that the matrix $\left(g^{l m}\right)$ is not diagonal, which means that the new coordinate system is nonorthogonal. Thus it seems natural to use Maxwell's equations under their tensor form that has been derived from relativistic electrodynamics. In this work, we will use Maxwell's equations under the covariant form introduced by Post. ${ }^{8}$

Working with linear, homogeneous, and isotropic media and assuming that time dependence is handled by the term $\exp (i \omega t)$, the complex amplitudes $E_{n}$ and $H_{n}$ of the covariant components of the electric and the magnetic fields are linked by the following Maxwell's equations:

$$
\begin{aligned}
\xi^{l m n} \partial_{m} E_{n} & =-i \omega \mu_{0} \sqrt{g} g^{l m} H_{m}, \\
\xi^{l m n} \partial_{m} H_{n} & =i \omega \varepsilon_{0} \nu^{2} \sqrt{g} g^{l m} E_{m}, \\
1 & \leqslant l, m, n \leqslant 3, \quad g=\operatorname{det}\left(g_{l m}\right) .
\end{aligned}
$$

$\varepsilon_{0}$ and $\mu_{0}$ are the dielectric permittivity and the magnetic permeability of vacuum, respectively; $\nu$ is the refractive index of the medium; and $\partial_{m}$ denotes the derivation operator with respect to variable $x^{m} \cdot \xi^{l m n}$ is the LeviCivita indicator defined by

$$
\xi^{l m n}=\left\{\begin{array}{ll}
1 & \text { if }(l m n) \text { is an even permutation of }(123) \\
-1 & \text { if }(l m n) \text { is an odd permutation of }(123) \\
0 & \text { otherwise }
\end{array} .\right.
$$

If we substitute the metric tensor ( $\left.g^{l m}\right)$ from Eq. (2) into Eqs. (3), and keeping in mind that the problem is $z$ invariant (i.e., $\partial_{3} \equiv 0$ ), we obtain

$$
\begin{aligned}
\partial_{2} E_{3} & =-i \omega \mu_{0}\left(H_{1}-\dot{a} H_{2}\right), \\
-\partial_{1} E_{3} & =-i \omega \mu_{0}\left(-\dot{a} H_{1}+\left(1+\dot{a}^{2}\right) H_{2}\right), \\
\partial_{1} E_{2}-\partial_{2} E_{1} & =-i \omega \mu_{0} H_{3}, \\
\partial_{2} H_{3} & =i \omega \varepsilon_{0} \nu^{2}\left(E_{1}-\dot{a} E_{2}\right),
\end{aligned}
$$

$$
-\partial_{1} H_{3}=i \omega \varepsilon_{0} \nu^{2}\left(-\dot{a} E_{1}+\left(1+\dot{a}^{2}\right) E_{2}\right),
$$

$$
\partial_{1} H_{2}-\partial_{2} H_{1}=i \omega \varepsilon_{0} \nu^{2} E_{3} .
$$

From these equations, it can be shown ${ }^{5}$ that the two types of polarization (TE and TM) are described by the same set of differential equations:

$$
\partial_{u} F(x, u)=d(x) \partial_{x} F(x, u)-i k c(x) G(x, u),
$$

$$
\begin{aligned}
\partial_{u} G(x, u)= & -\frac{i}{k} \partial_{x}\left[c(x) \partial_{x} F(x, u)\right]-i k F(x, u) \\
& +\partial_{x}[d(x) G(x, u)],
\end{aligned}
$$

where $\quad c(x)=1 /\left(1+\dot{a}^{2}\right), \quad d(x)=\dot{a} /\left(1+\dot{a}^{2}\right), \quad$ and $\quad k=k_{0} \nu$ $=\omega \sqrt{\epsilon_{0} \mu_{0}} \nu$. The TE case corresponds to $F=E_{z}$ and $G$ $=Z H_{x}$ whereas the TM case corresponds to $F=Z H_{z}$ and $G=-E_{x}$ with $Z=\sqrt{\left(\mu_{0} / \epsilon_{0}\right)} / \nu=Z_{0} / \nu$.

Due to the $x$ periodicity, $c(x)$ and $d(x)$ can be expanded in their Fourier series whereas $F(x, u)$ and $G(x, u)$ are expanded in generalized Fourier series:

$$
\begin{aligned}
c(x)= & \sum_{m} c_{m} \exp (-i m 2 \pi x / d), \quad d(x)=\sum_{m} d_{m} \exp ( \\
& -i m 2 \pi x / d), \\
F(x, u)= & \exp \left(-i \alpha_{0} x\right) \sum_{m} F_{m}(u) \exp (-i m 2 \pi x / d), \\
G(x, u)= & \exp \left(-i \alpha_{0} x\right) \sum_{m} G_{m}(u) \exp (-i m 2 \pi x / d) .
\end{aligned}
$$

Here $\alpha_{0}$ is a real number (often called the quasiperiodicity factor) that stands for the $x$ component of the incident wave vector. Introducing these expressions in Eqs. (7) and projecting on the Fourier basis, gives

$$
\frac{\mathrm{d}}{\mathrm{d} u}\left[\begin{array}{l}
F_{m} \\
G_{m}
\end{array}\right]=-i\left[\begin{array}{ll}
{[|d|] \alpha} & k[|c|] \\
k-\frac{1}{k} \alpha[|c|] \alpha & \alpha[|d|]
\end{array}\right]\left[\begin{array}{c}
F_{m} \\
G_{m}
\end{array}\right],
$$

$\alpha=\operatorname{diag}\left(\dot{\alpha}_{0}+m 2 \pi / d\right), \quad[|c|]=(1+[|\dot{a}|] \times[|\dot{a}|])^{-1}, \quad[|d|]=[|c|]$ $\times[|\dot{\alpha}|]$, and $[|\dot{\alpha}|]_{m n}$ is the $(m-n)$ th Fourier coefficient in the development of $\dot{a}(x)$. It is worth noting that the correct rules of Fourier factorization ${ }^{9}$ have been used. Equation (9) can be written under the more concise form

$$
\frac{\mathrm{d} \mathcal{F}}{\mathrm{d} u}=\mathcal{A} \mathcal{F}(u), \quad \mathcal{F}=\left[\begin{array}{c}
F_{m}(u) \\
G_{m}(u)
\end{array}\right] .
$$

$\mathcal{A}$ is the matrix appearing in Eq. (9). At this stage we truncate the differential system by keeping only the orders such that $-M \leqslant m \leqslant M$ ( $M$ will be called the truncation order). Then, assuming a $u$ dependence in exponential form, the above differential system can be transformed into an eigenvalue problem:

$$
r_{n} \mathcal{F}_{n}=\mathcal{A} \mathcal{F}_{n},
$$

where $r_{n}$ is an eigenvalue of $\mathcal{A}$, and $\mathcal{F}_{n}$ is its corresponding eigenvector. At this stage and to distinguish between propagating and evanescent waves and between those propagating toward the grating and those traveling to in- 
finity, the eigenvalues are sorted according to their real and imaginary parts. ${ }^{5}$ Finally, the fields corresponding to our diffraction problem can be written

medium 1:

$$
\begin{aligned}
F_{1}(x, u)= & \sum_{m=-M}^{M}\left\{F_{m 0}^{i n c} \exp \left(r_{10} u\right)+\sum_{n=1}^{2 M+1} R_{n} F_{m n}^{\text {diff }} \exp \left(r_{1 n} u\right)\right\} \\
& \times \exp \left(-i \alpha_{m} x\right) \\
G_{1}(x, u)= & \sum_{m=-M}^{M}\left\{G_{m 0}^{i n c} \exp \left(r_{10} u\right)+\sum_{n=1}^{2 M+1} R_{n} G_{m n}^{\text {diff }}\right. \\
& \left.\times \exp \left(r_{1 n} u\right)\right\} \exp \left(-i \alpha_{m} x\right)
\end{aligned}
$$

medium 2:

$$
\begin{aligned}
& F_{2}(x, u)=\sum_{m=-M}^{M}\left\{\sum_{n=1}^{2 M+1} T_{n} F_{m n}^{t r} \exp \left(r_{2 n} u\right)\right\} \exp \left(-i \alpha_{m} x\right), \\
& G_{2}(x, u)=\sum_{m=-M}^{M}\left\{\sum_{n=1}^{2 M+1} T_{n} G_{m n}^{t r} \exp \left(r_{2 n} u\right)\right\} \exp \left(-i \alpha_{m} x\right),
\end{aligned}
$$

where $r_{10}$ is the eigenvalue corresponding to the incident plane wave while $r_{1 / 2 n}$ corresponds to the reflected (propagating+evanescent) or transmitted (propagating + evanescent) waves in mediums 1 and 2 , respectively. $R_{n}$ and $T_{n}$ are the reflection and the transmission amplitudes to be determined via the boundary conditions. As mentioned in Section 1, we will use the CBCM, and therefore it is useful to give an idea about the principle of this method.

\section{PRINCIPLE OF THE COMBINED BOUNDARY CONDITIONS METHOD}

In this section, we briefly recall the principle of the CBCM for flat strip gratings. The interested reader can find detailed developments in Refs. 1, 2, and 10. The situation is that of Fig. 1 with a null height. The boundary conditions at the interface $y=0$ impose that

- the tangential electric field must be continuous over a whole period;

- the tangential electric field must be null over the strips; and

- the magnetic field must be continuous over the complementary of the strips.

The main idea behind the CBCM is to combine the last two conditions in a single one that is valid over a whole period. The benefit is that one can use the orthonormal Fourier basis $\left[\exp \left(-i \alpha_{m} x\right)\right]$ as projection one.

In the TE polarization case, these conditions write

$$
\begin{aligned}
& E_{1 z}(x, y)=E_{2 z}(x, y) \text { for } 0<x<D, \\
& E_{1 z}(x, y)=E_{2 z}(x, y)=0 \text { for } 0<x<w,
\end{aligned}
$$

$$
H_{1 x}(x, y)=H_{2 x}(x, y) \text { for } w<x<D,
$$

and can be recast, as mentioned above, to the following set of two relations:

$$
\begin{aligned}
\forall 0 & <x \\
& <D\left\{\begin{array}{l}
E_{1 z}(x, y)=E_{2 z}(x, y) \\
\chi(x) E_{2 z}(x, y)+\tau[1-\chi(x)]\left[H_{2 x}(x, y)-H_{1 x}(x, y)\right]=0,
\end{array}\right.
\end{aligned}
$$

where $\chi(x)$ is the characteristic function of the strips, i.e., $\chi(x)=1$ over the strips and zero elsewhere. $\tau$ is a numerical parameter introduced for dimensional and numerical purpose: As we mix boundary conditions involving the electric and the magnetic fields, that are of different magnitudes, a problem of bad numerical conditioning of the matrices can arise. This is avoided by introducing the parameter $\tau$ that can be adjusted in such a way that the two terms constituting the mixed equation are of the same scale. In principle the second equation, in Eq. (15) is valid whatever the value of $\tau$ (see Ref. 1, for example).

In the TM polarization case, the boundary conditions write

$$
\begin{aligned}
& E_{1 x}(x, y)=E_{2 x}(x, y) \quad \text { for } 0<x<D, \\
& E_{1 x}(x, y)=E_{2 x}(x, y)=0 \text { for } 0<x<w, \\
& H_{1 z}(x, y)=H_{2 z}(x, y) \quad \text { for } w<x<D,
\end{aligned}
$$

and can be recast to

$$
\begin{aligned}
\forall 0 & <x \\
& <D\left\{\begin{array}{l}
E_{1 x}(x, y)=E_{2 x}(x, y) \\
\chi(x) E_{2 x}(x, y)+\tau[1-\chi(x)]\left[H_{2 z}(x, y)-H_{1 z}(x, y)\right]=0
\end{array} .\right.
\end{aligned}
$$

Thus writing the boundary conditions through the concept of the CBCM consists of using Eqs. (15) in the TE case and Eqs. (17) in the TM case. Now we are going to apply this principle to the curved strip grating described in Section 1. For that purpose it is convenient to separate the two types of polarization.

\section{TE POLARIZATION}

In this case, and by use of the CBCM, the boundary conditions on the surface $y=a(x)$ are expressed through Eqs. (15), which give, in the translation coordinates system, on the surface that corresponds to $u=0$ :

$$
\begin{aligned}
& \forall 0<x \\
& \quad<D\left\{\begin{array}{l}
F_{1}(x, u=0)=F_{2}(x, u=0) \\
\chi(x) F_{2}(x, 0)+\tau[1-\chi(x)]\left[\frac{1}{Z_{2}} G_{2}(x, 0)-\frac{1}{Z_{1}} G_{1}(x, 0)\right] \\
=0 .
\end{array}\right.
\end{aligned}
$$


Expanding the characteristic function in Fourier series and projecting the above equations on the exponential basis gives, in vector notation,

$$
\begin{gathered}
\mathbf{F}^{\text {inc }}+F^{\text {diff }} \mathbf{R}=F^{\text {tr }} \mathbf{T} \\
{[|\chi|] F^{t r} \mathbf{T}+\frac{\tau}{Z_{0}}[\mathbf{I}-[|\chi|]]\left\{\nu_{1} G^{t r} \mathbf{T}-\nu_{2}\left(\mathbf{G}^{i n c}+G^{\text {diff }} \mathbf{R}\right)\right\}=0 .}
\end{gathered}
$$

I stands for the identity matrix and $[|\chi|]$ is the toeplitz matrix whose $(m, n)$ th elements are the Fourier coefficients $\chi_{m-n}$. The rest of the work is rather mechanical. The linear system of Eqs. (19) is solved for $\mathbf{R}$ and $\mathbf{T}$ from which the fields can be calculated everywhere.

\section{TM POLARIZATION}

In this second case, the boundary conditions on the surface defined by $u=0$, in the translation coordinates system, write

$$
\begin{aligned}
\forall 0<x & \\
& <D\left\{\begin{array}{l}
G_{1}(x, u=0)=G_{2}(x, u=0) . \\
\chi(x) G_{2}(x, 0)+\tau[1-\chi(x)]\left[F_{2}(x, 0)-F_{1}(x, 0)\right]=0
\end{array}\right.
\end{aligned}
$$

By expanding the characteristic function in Fourier series and projecting the above equations on the exponential basis, we obtain in vector notations

$$
\mathbf{G}^{\text {inc }}+G^{\text {diff }} \mathbf{R}=G^{\text {tr }} \mathbf{T}
$$

$$
[|\chi|] G^{t r} \mathbf{T}+\frac{\tau}{Z_{0}}[\mathbf{I}-[|\chi|]]\left\{\nu_{1} F^{t r} \mathbf{T}-\nu_{2}\left(\mathbf{F}^{i n c}+F^{\text {diff }} \mathbf{R}\right)\right\}=0
$$

As in the TE case, the linear system of Eqs. (21) is solved for $R$ and $T$ from which the fields can be calculated everywhere.

\section{EFFICIENCIES}

Finally, application of Poyntings theorem, written in the translation coordinates, enables us to calculate the power carried by the $n$th diffracted and transmitted waves. Diffraction efficiencies are then defined as the ratio of these powers to that carried by the incident wave ${ }^{5}$ :

$$
\begin{array}{r}
e r_{n}=\left|R_{n}\right|^{2} \frac{\operatorname{Re}\left(\sum_{m=-M}^{M} F_{m n}^{\text {diff }} G_{m n}^{\text {diff }}\right)}{\operatorname{Re}\left(\sum_{m=-M}^{M} F_{m 0}^{i n c} G_{m 0}^{i n c}\right)}, \\
e t_{n}=\frac{\nu_{2}}{\nu_{1}}\left|T_{n}\right|^{2} \frac{\operatorname{Re}\left(\sum_{m=-M}^{M} F_{m n}^{t r} G_{m n}^{t r}\right)}{\operatorname{Re}\left(\sum_{m=-M}^{M} F_{m 0}^{i n c} G_{m 0}^{i n c}\right)} .
\end{array}
$$

\section{RESULTS}

In this section, we provide some numerical results to illustrate the effectiveness of the presented method. Let us first emphasize that the convergence of the method has been checked against usual criteria such as energy conservation (up to $10^{-9}$ ) and reciprocity laws.

As a first example we consider a sinusoidal profile described by the function $a(x)=h / 2 \cos (2 \pi x / D)$. We begin by setting $h=0$, and verify that we recover the results obtained from the classical CBCM in the case of planar structures as published in Ref. 10 . The period is such that $\lambda=0.7 D$, the gap between the slits is $w=D / 2$, the incidence angle is $\theta=26^{\circ}$ while the surrounding medium is vacuum. The truncation order has been taken equal to 256 and $\tau$ has been set to $0.01 i$. In Table 1 we give our computed reflected and transmitted efficiencies for both cases of polarization with $h=0$. These results match those of Ref. 10.

We now set the height of the grating to $D / 2$ (all the other parameters being unchanged) and observe the reflectivity (sum of all the reflected efficiencies) of the structure versus the parameter $w$. Figure 2 demonstrates the change in reflectivity when the planar strips are bent. The case of TE polarization is remarkable because of the quick variation in reflectivity that appears near $w$ $=0.75 D$, which approximately corresponds to the wavelength. This could be explained by the formation of a cavity corresponding to the lower part of the cosine function.

In the second example, and for the purpose of validation, we compare our results to those obtained by an integral approach. ${ }^{11}$ The profile is trapezoidal as depicted in Fig. 3 and the metal is deposited along the lateral sides of the trapezium. As can be seen from Fig. 4, where the reflected efficiencies are drawn as functions of the parameter $D / \lambda$, our results coincide exactly with those of Ref. 11. Furthermore, we verified that by setting the strips on

Table 1. Computed Diffraction Efficiencies for $h=0$

\begin{tabular}{cr}
\hline TE & TM \\
\hline$e r_{-2}=0.0256$ & $e r_{-2}=0.0257$ \\
$e r_{-1}=0.1799$ & $e r_{-1}=0.1790$ \\
$e r_{0}=0.3707$ & $e r_{0}=0.2212$ \\
$e t_{0}=0.2183$ & $e t_{0}=0.3694$ \\
\hline
\end{tabular}
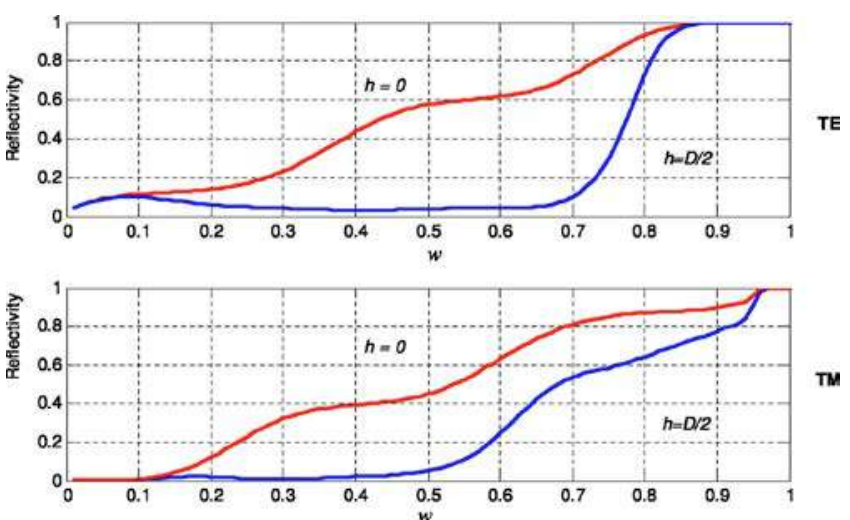

Fig. 2. (Color online) Reflectivity as a function of the width $w$ of the strips. 


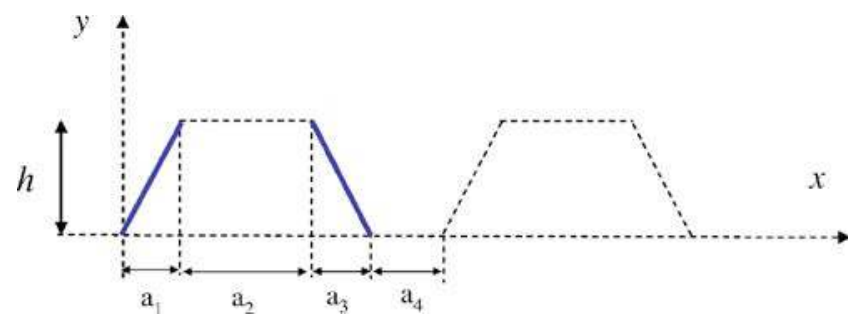

Fig. 3. (Color online) Schematic view of the trapezoidal grating used in example 2. $D=\sum_{i=1,4} a_{i}$ with $a_{2}=a_{4}=D / 3$ and $a_{1}=a_{3}$ $=D / 6=h \tan (\pi / 6)$. The angle of incidence is set to $0.1^{\circ}$.

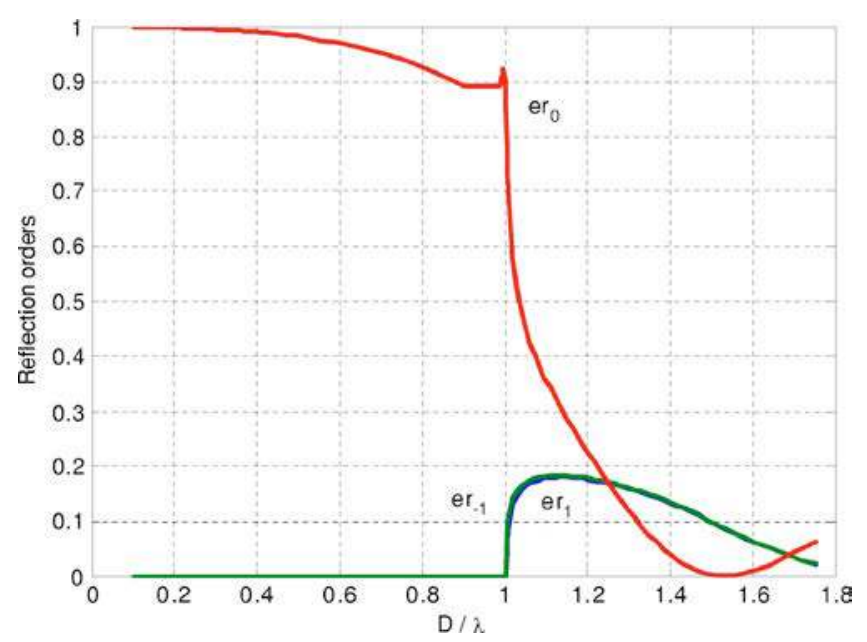

Fig. 4. (Color online) Evolution of the different reflected efficiencies $e r_{(-1,0,1)}$ versus $D / \lambda$.
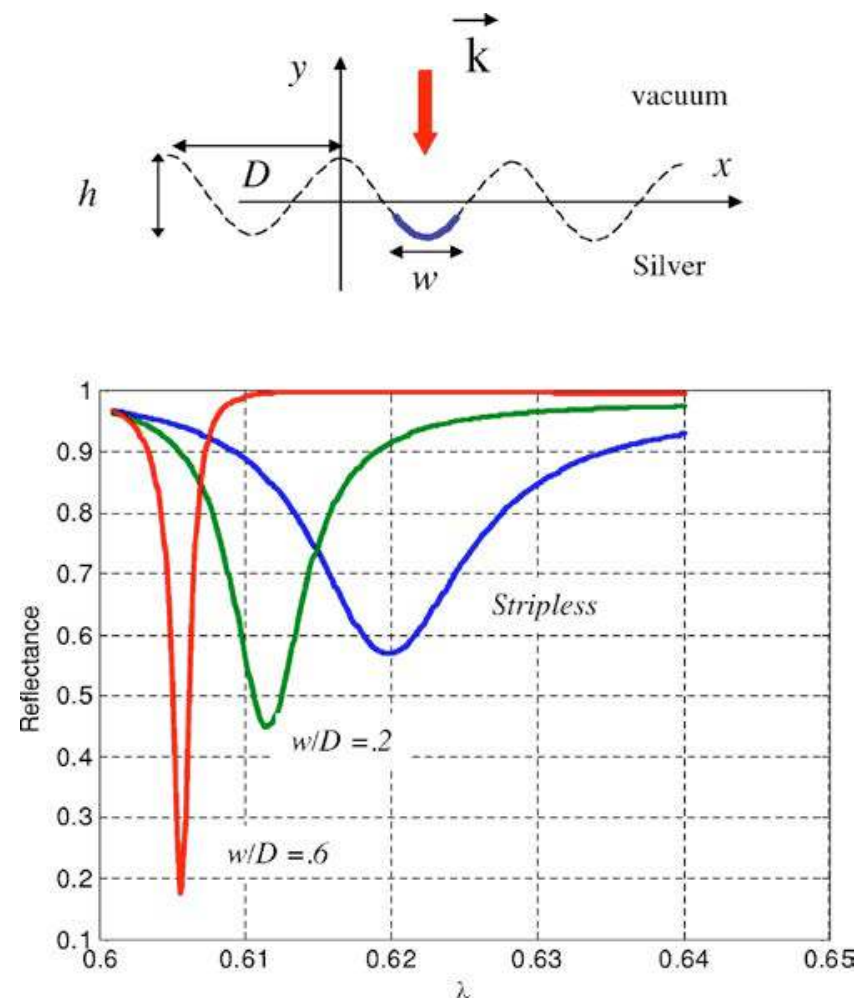

Fig. 5. (Color online) Reflectivity versus the wavelength for a metallic sinusoidal grating covered with strips of various widths. $h=0.05 \mu \mathrm{m}, D=0.6 \mu \mathrm{m}, \epsilon_{\text {sil }}=-17.6+0.67 j$. one horizontal part of the trapezium we recover the results computed by use of the classical planar CBCM.

As an application, we consider a metallic (silver) sinusoidal grating that can support a surface plasmon. Let us imagine now an academic experience in which this structure is partially covered by a strip grating and illuminated by a monochromatic plane wave under TM polarization. In this example, we neglect the dispersion and set $\epsilon_{\text {sil }}=-17.6+0.67 j$. Figure 5 shows the evolution of the calculated reflectivity versus the wavelength for various widths $w$ of the strips. We notice that as the width of the strips is increased, the dip in reflection that is characteristic of the excitation of a surface plasmon is shifted toward the lower part of the spectrum and at the same time becomes more and more sharp. This behavior is due to the fact that the presence of the strips affects the dispersion relation of the original silver grating that is responsible for the shift of the plasmon resonance. The fineness of the dips can be easily understood if one keeps in mind that their width is directly related to the losses in the metal (silver). As the lossy metal is covered by the perfectly conducting strips that are lossless, the amount of losses is reduced and thus the resonances are sharper. A more detailed study of this interesting phenomenon is in progress that introduces the real dispersion of silver in the model and computes the map of the fields around the strips.

\section{CONCLUSION}

A method has been presented that combines two of the most efficient known methods in the field of diffraction grating theories. We showed that this new approach is general in the sense that it allows the study of curved strip gratings of various shapes with a rather low computational effort. Furthermore, numerical examples were given with tabulated values that can be useful for those who want to check their codes.

Finally, let us emphasize that the method can be easily adapted for the study of multilayered strip structures and can be extended, without great difficulty, to crossed curved gratings.

B. Guizal's e-mail address is bguizal@univ-fcomte.fr.

\section{REFERENCES}

1. F. Montiel and M. Nevière, "Electromagnetic study of the diffraction of light by a mask used in photolithography," Opt. Commun. 101, 151-156 (1993).

2. F. Montiel and M. Nevière, "Perfectly conducting gratings: a new approach using infinitely thin strips," Opt. Commun. 144, 82-88 (1997).

3. B. Guizal and D. Felbacq, "Electromagnetic beam diffraction by a finite strip grating," Opt. Commun. 165, 1-6 (1999).

4. B. Guizal and D. Flebacq, "Numerical computation of the scattering matrix of an electromagnetic resonator," Phys. Rev. E 66, 026602 (2002).

5. J. Chandezon, M. T. Dupuis, G. Cornet, and D. Maystre, "Multicoated gratings: a differential formalism applicable in the entire optical region," J. Opt. Soc. Am. 72, 839-846 (1982).

6. G. Granet, J. P. Plumey, and J. Chandezon, "Scattering by a periodically corrugated dielectric layer with non-identical faces," Pure Appl. Opt. 4, 1-5 (1995).

7. G. Granet, "Analysis of diffraction by crossed gratings 
using a non-orthogonal coordinate system," Pure Appl. Opt. 4, 777-793 (1995).

8. E. J. Post, Formal Structure of Electromagnetics (North Holland, 1962).

9. L. $\mathrm{Li}$ and J. Chandezon, "Improvement of the coordinate transformation method for surface-relief gratings with sharp edges,” J. Opt. Soc. Am. A 13, 2247-2255 (1996).
10. G. Granet and B. Guizal, "Analysis of strip gratings using a parametric modal method by Fourier expansions," Opt. Commun. 255, 1-11 (2005).

11. R. C. Hall, R. Mittra, and K. M. Mitzner, "Scattering from finite thickness resistive strip gratings," IEEE Trans. Antennas Propag. 36, 504-510 (1988). 